\title{
A Contextualised Learning Interaction Memory
}

\author{
Sandra A. Siebra ${ }^{1,2}$, Ana Carolina Salgado $^{1}$ \& Patrícia A. Tedesco ${ }^{1}$ \\ ${ }^{1}$ Centro de Informática - UFPE \\ Caixa Postal 7851 - Cidade Universitária, - CEP 50732-970 Recife - PE - Brasil \\ ${ }^{2}$ Faculdade Integrada do Recife (FIR) \\ Av. Abdias de Carvalho, 1678 - Madalena - CEP: 50720-635 Recife - PE - Brasil \\ \{sas, acs, pcart\}@cin.ufpe.br
}

\begin{abstract}
The interaction is the key element used in Collaborative Learning Environments to understand the process of knowledge building and the role played by each student in it. Interaction analysis can provide support for the students' reflection and self-regulation processes as well as for the teachers' activities. But to perform the analysis process, it is important to discover and register the context where each interaction has occurred, in order to understand the meaning of user interactions. However, although there are several approaches for Interaction Analysis in Computer-Supported Collaborative Learning, there is a lack of methods and tools that consider: (1) the context where the interactions have occurred; (2) the different needs of feedback from the point of view of teacher and students; and (3) the necessity of contextualized historical information to produce more complete and semantically rich reports for students and teachers. In this light, this paper presents a Learning Interaction Memory (LIM), used to store the learning interactions occurred in Computer-Supported Collaborative Learning Environments, taking into account the context where they have occurred. The LIM was modelled in a multidimensional structure so that interactions can be viewed from different perspectives and can be presented selectively, according to users' needs. This paper also presents the process of construction and exploration of the LIM and a Context-Based Analytical Environment called SmartChat + : an environment for collaborative discussions
\end{abstract}

of specific subjects that uses the LIM. In order to show the feasibility of the LIM, a series of exploratory studies was carried out, yielding good initial results, especially in what concerns feedback from the users.

Keywords: Interaction Analysis, Collaborative Learning, Group Memory, Context, CSCLE.

\section{INTRODUCTION}

Collaborative Learning is a strategy in which small teams, each with students of different levels of ability, use a variety of learning activities to improve their understanding of a subject. Each member of a team is responsible not only for learning what is taught but also for helping teammates learn, thus creating an atmosphere of achievement and collaboration [14]. Computer-Supported Collaborative Learning (CSCL) applications for online collaborative learning are characterized by a high degree of user-user and user-system interaction and hence generate a huge amount of information.

In general, CSCLE (Computer Supported Collaborative Learning Environments) can offer greater opportunities to share and solicit knowledge through the interaction. In most situations, externalization, articulation, argumentation, negotiation of multiple perspectives, are considered to be the main mechanisms that can promote collaborative learning [44][47]. When learners work in groups they reflect upon their ideas (and those of their colleagues), explain their opinions, consider and discuss those of 
others, and as a result, they learn. In this way, each learner acquires individual knowledge from the collaborative interaction. In this light, interactions taking place in CSCLE are one of the most important visible aspect of collaboration and the analysis of these interactions could provide information to teachers (so that they are able to follow the evolution of students in the short, medium and long terms) and to students so that they can improve their knowledge and reflect about it. However, in most current CSCLE there is a lack of proper mechanisms to store and retrieve past interactions. Thus it is much more difficult for group members (and instructors) to retrieve knowledge that has already been shared. This may hinder the learning process - members miss an opportunity for reflection - and the evaluation process - instructors have a harder time carrying out continuous, diagnostic evaluation of the group development.

In this state of affairs, a basic requirement to support the analysis of collaborative interactions is to provide an adequate persistence mechanism to store the interactions. Without persistence, interaction is ephemeral and cannot be shared afterwards with people who were not involved at the time it occurred [4]. With an adequate persistence means, the interactions will constitute what we call Learning Interaction Memory (LIM), which makes historical information about the interactions readily and selectively available when required. Thus, the LIM should be modelled in such way that stored interactions can be viewed from different perspectives (e.g. information can be easily crossed or filtered) and presented selectively, according to users' needs (i.e., depending on their context, users could access different information). Indeed, the LIM's model should facilitate the interaction analysis as well as to provide access to the information and construction and generation of customized reports to teachers and students.

Other point to consider when constructing a complete historical model to store the learning interactions is to capture and store the context where each interaction occurred [6]. Context is a collection of relevant conditions and surrounding influences that make a situation unique and comprehensive [5]. In many cases, only the context can provide the complete understanding of many interactions, actions or events and the correct cues that allow the CSCLE to correctly analyse the interactions in order to better support user reflection and to provide adequate feedback [1][33]. Through the analysis of the contextualized information stored in the LIM, it is possible to characterize interactions for a better understanding of the collaborative learning process.

In this light, this paper proposes the creation of the LIM to store the learning interactions occurred in CSCLE. The
LIM takes into account contextual information (to enrich the stored knowledge) and is modelled as a multidimensional repository. As a result, the interactions can be explored in different dimensions and levels of detail, and specific feedback can be provided to both teachers and students (an advantage over common log files). This paper also presents a summary of a process for the construction and exploration of the LIM and an environment for collaborative discussions of specific subjects that uses the LIM, together with the initial exploratory studies carried out with this environment. The results of the exploratory studies indicate the feasibility of this kind of memory to support effective learning. The contextualization of the interactions and the following creation of the LIM are parts of a complete context-based process for interaction analysis proposed by Siebra and colleagues [36] which is summarized in Section 3 of this paper.

The remainder of this document is structured as follows: Section 2 discusses the importance of the LIM and present some related work. Section 3 briefly describes the context-based process for interaction analysis in which the LIM is one of the generated artefacts. Section 4 discusses the semantic enrichment of the LIM through interaction contextualizing. Section 5 presents the modelling and creation of the LIM. Section 6 discusses the implementation issues related to SmartChat + , a context-based analytical environment that uses the LIM. Section 7 shows the exploratory studies carried out with SmartChat + and discusses their results. Finally, section 8 presents our conclusions and suggestions for further work.

\section{The Importance OF THE Learning INTERACTION MEMORY}

One of the key elements of success in group work is the use of an organizational memory $(\mathrm{OM})$ that stores the knowledge gained from past work of the group [35]. The $\mathrm{OM}$ was defined as "stored information from an organizations' history that can be brought to bear on present decisions" [49]. By keeping track of past solutions to organizational problems as well as the rationale behind past decisions, organizations can avoid wasting time, money and effort. The OM can facilitate group learning, provide justification for group decisions and improve the efficiency of group decision makers. A type of organizational memory is the group memory, which can be used in various group tasks such as, meetings, trainings and projects. Satzinger, Garfield and Nagasundaram [34] describe how contents of group memory in Group Support Systems influence ideas generated by individuals. Kantor and colleagues [22] discuss how to provide awareness for the group members using the information in the group memory. 
Nowadays, there are several proposals of CSCLE (e.g. [17][19][44]) that provide a wide variety of interaction mechanisms such as forums, blackboards, chats, email and videoconferences. However, one of the main problems with the use of interaction mechanisms in CSCLE is the frequently inappropriate match between the interaction mechanism available and the persistency required [4]. Group members may find it difficult to recall and justify their decisions when using interaction mechanisms with low or no persistence. Important information may be lost or needed to be reproduced several times so that the group can achieve a desirable level of common knowledge [4].

While researching CSCLE that use group memories as a source of information to generate feedback for students and teachers, we have analysed some collaborative environments and the evaluation processes used for them (e.g. [9][11][19][25][31][43][44][48]). We have observed that most of the environments have inadequate mechanisms for interactions' persistence - group members may find it difficult to recall and justify their decisions when using interaction mechanisms with low or no persistence. Although most of them provide a way to store previously sent or received messages (e.g., by using sequential log files, normally organised in temporal order or in XML hierarchies), what it is really needed is a group memory to store the information. Thus, it would be possible to comfortably refer to it, add new contributions and analyse the stored information, according to the user's needs. Such a group memory would be an invaluable source for evaluating the collaborative process.

Other issue to consider is the absence of the context of the information in the group memory. A barrier to the effective use of group memories is that the usual approach to building group memories based only on preserving information, fails to preserve the context which gives the information meaning, the very thing that allows them to be useful in the future, when the context has changed [8]. All the collaborative environments analysed neglect the context where the interactions occur when they create the interaction history. However, the identification of the contextual elements is relevant to characterise, enrich and qualify the interactions. Moreover, identifying the context in which the interactions occurred is fundamental to extract the knowledge produced in them. In that respect, the method proposed by Daradoumis et al. [11] is an exception - they consider both qualitative and quantitative aspects of the collaborative interaction. In performing Social Network Analysis, they consider part of the context where the interactions were produced.

Furthermore, we have observed that most environments do not use the stored information to generate any feedback or reports for students and teacher and even the systems that provide feedback do so through reports or statistics generated from the interaction log, and only at the end of the discussion. There is no feedback provided during the discussion (in that respect, the OXEnTCHÊ [48] is an exception).

In brief, although many CSCLE provide a way to store previously sent or received messages (e.g., by using sequential $\log$ files, normally organised in temporal order), what is really needed is a shared space to store the information in order to comfortably refer to it and add new contributions. This shared space is the LIM. Thus, the LIM is the record of the complete group interaction process. It is the result of a process of accumulating data generated by group members during discussions in synchronous and asynchronous tools. The LIM is an important resource in collaborative dialogue since it provides a common reference to previous activity (unlike most spoken dialogues) that may encourage reflection and more effective collaboration [7]. This kind of information could help users to reuse historical information to solve future problems, reminding participants of previous ideas (encouraging elaboration on them) and possibly serving as an agenda for further work. By exploring the LIM, teachers will have access to information that supports a continuous evaluation of their students, their learning strategies and the lessons' content, and they can get more indications about the proper moment to interact and motivate their students. All of these capabilities can significantly improve the potential success of computer based learning processes. Additionally, using the information stored in the LIM, teachers/systems can better decide which one is the adequate feedback for a given situation.

In order to generate the LIM, it is necessary to consider two fundamental issues: the collaborative interactions (type and quantity) and the context in which they have occurred. Below we present a Context-Based Process for Interaction Analysis that produces the LIM and guides its exploration. When presenting the process, we will focus on the stages that are directly related to the production of the LIM.

\section{A Context-BASEd Process FOR INTERACTION ANALYSIS}

In order to analyse user interactions so that we can support the students' reflection process and the teacher in her/his activities, we have developed a four-stage Contextbased Process for Interaction Analysis ${ }^{1}$ (see Figure 1).

\footnotetext{
${ }^{1}$ All figures of the process were made using the SPEM (Software Process Engineering Metamodel) notation [45].
} 


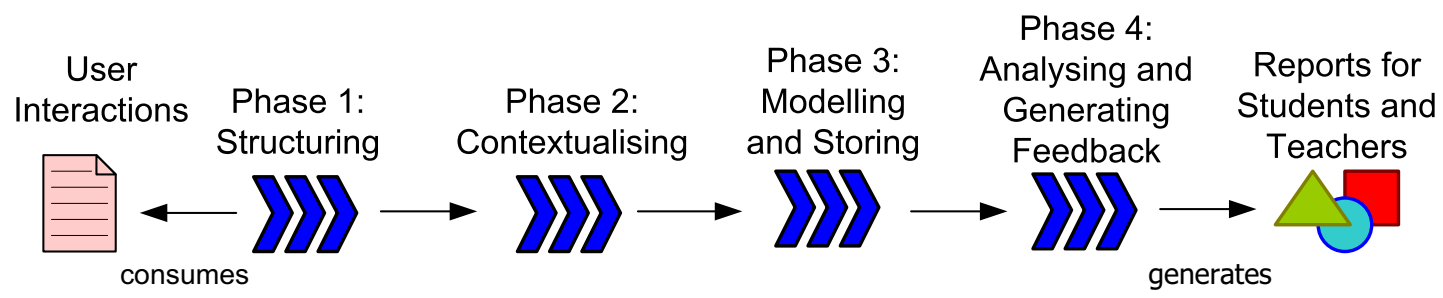

Figure 1: Stages of the Context-Based Process for Interaction Analysis

Stage 1: Structuring is the first stage of process because it makes the intention of the exchanged messages and the relations among them explicit. In this stage, the input is all messages (User Interactions) exchanged by users in the chat environment. The stage objective is the categorisation (either automatic or user-provided) and connection of the messages using an argumentation model. In order to structure the interactions, we have proposed an Argumentation Model for collaborative discussions [38] based on the IBIS (Issue Based Information System) Argumentation Model [24] to classify and relate the interactions. The output of this stage is a discussion graph.

Stage 2: Contextualising is the second stage of the process. In this stage, the input is the Discussion Graph generated previously. The stage objective is to enrich each interaction in the Discussion Graph with contextual information and to create a Contextual Repository with the contextual information that could be useful to the interaction analysis (e.g., Subject, Group and Individual contexts). This contextual information can be captured implicitly (using monitoring agents or sensors) or explicitly (through questionnaires or forms filled in by the users). The outputs are the Contextualized Interactions (interactions with the context required to better understand them - just a part of the contextual information such as Interaction, Individual, Subject and Session contexts) and the Contextual Repository (that keeps all the contextual information, captured by the environment and that is required during the analysis stage).

Stage 3: Modelling and Storing. In this stage the input is the Contextualised Interactions and the Contextual Repository that were generated previously. The stage objectives are twofold: modelling the contextualised interactions in a multidimensional schema [23] and storing these interactions in a multidimensional repository. The output is the LIM.

Stage 4: Analysing and Generating Feedback. In this last stage the input is the information in the LIM that are accessed using Analytical Queries. There are two stage objectives: using Analytical Queries to explore the LIM and, based on the query's answers, generating feedback for students and teachers according to their needs. The outputs are reports for students and teachers.

This paper focuses on stages 2 (Contextualising) and 3 (Modelling and Storing) of this process because the main part of these stages are relate to the construction of the LIM. As we have already argued, without the LIM, it will not be possible generate feedback for students and teachers (the goal of the interaction analysis process).

\section{ENRICHING THE INFORMATION IN THE}

\section{LIM: INTERACTION CONTEXTUALISING}

Context is a complex description of the knowledge shared on physical, social, historical and other circumstances where actions or events happen in the real world [5]. Although this knowledge is not part of the actions being executed or the events that occur, it will constrain the execution of an action or event interpretation without explicitly intervening in it [5].

Several domains $[3][6][10][13][30]$ have already elaborated their own working definition of context. Although all the definitions are somewhat different, it is commonly agreed that context is about evolving, structured and shared information spaces, and that such spaces are designed to serve a particular purpose [10]. Bazire and Brézillon [3] show that all the definitions found on the web can be assembled around six questions:

- Who? - Information about people;

- What? - Information about users activities in progress;

- When? - Information about time and historical Information;

- Where? - Information about environment; 
- Why? - Information about the reasons related to the user actions;

- How? - Information about user's action plans.

In conversation, context plays a fundamental role in disambiguating utterances [5]. Group members may find it difficult to recall information about the discussion, to justify their decisions when using interaction mechanisms, to remember previous ideas or to understand what other users have said when they do not know the context of each interaction. In many cases, only the context can provide the correct cues to give the right interpretation to a sentence or an action. In situations where geographically distributed individuals have to collaborate (especially if they are interacting asynchronously), technological support for understanding and storing the contextual elements involved (e.g., location and users' goals) is very important.

In this scenario, in order to contextualize the interactions, we have identified different types of contexts at different levels, trying to include all the elements related to CSCLE that can help in the interaction analysis process and trying to answer five (Who? What? When? Where? How?) of the six questions mentioned by [3]. In our work, we have not worried about the question Why because in fact this is the most difficult question to answer. Indeed, it will be necessary to use some kind of Knowledge Discovery and Data Mining (KDD) to answer this question and, at this moment, we are only worried about how to extract information from the existent data. Thus, based on the generic conceptual framework for analysing the use of context in groupware, proposed by Rosa, Borges and Santoro [32], we have organised context in five different categories [39][40], summarised in Table 1 and described as follows. This organization is useful to map out the information that must be captured in the CSCLE to characterize each interaction.

Information about people and groups (Individual and Group Contexts) - The knowledge about the characteristics of individuals and the group as a whole is a resource that can be used by teachers to encourage interaction and collaboration [27]. This category includes:
a)Group Context - it is important to have some knowledge about the group (abilities, experience, etc) in the LIM and which users compose the LIM in some specific moment. This kind of information could help to analyse if the group works well and, maybe, can help to create new groups in the future;

b)Individual Context - the elements in this category help to characterise the user, as well as let other users to better understand her/his doubts, difficulties and actions in the environment. Some of the contextual information in this category can be obtained from the user's model (generally present in the CSCLE).

Information about scheduled tasks (Subject and Task Contexts) - in CSCLE it is important to know what the users are doing and which subject they are currently studying/discussing. So, this category includes:

a) Subject Context - it consists of information about the subject that users are currently discussing;

b) Task Context - in CSCLE several tasks are possible (for example, to study a lesson, do exercises, do tests, discuss about a subject or draw something). The elements in this category are necessary to keep information about these tasks in order to identify what an individual or group is or was doing.

Information about tasks and activities already concluded (History Context) - the information in this category tries to characterise the interactions that have already occurred. Its goal is to provide background information about the experiences learned either from the same group or from similar tasks performed by other groups. In this category, all contextual information generated is stored for future retrieval. It is the repository of the "group memory" (including contextual elements). Hence, a situation can be reconstructed with the context in which it occurred. This can also be used to share the latest news, seek advice and compare notes. Moreover, it allows students and/or teachers to access past events. Thus, it is a potential source of reflection for both the teacher and the student. 
Table 1: Conceptual Framework for Interaction Analysis Context

\begin{tabular}{|c|c|c|c|c|}
\hline $\begin{array}{l}\text { Related } \\
\text { Question }\end{array}$ & Information Type & $\begin{array}{l}\text { Associated } \\
\text { Contexts }\end{array}$ & \multicolumn{2}{|c|}{ Some Examples of Contextual Elements } \\
\hline \multirow[b]{2}{*}{ Who? } & \multirow[b]{2}{*}{ Group Members } & Group & $\begin{array}{l}\text { - Name } \\
\text { - Members } \\
\text { - Aim }\end{array}$ & $\begin{array}{l}\text { - Roles } \\
\text { - Group Manager }\end{array}$ \\
\hline & & Individual & $\begin{array}{l}\text { - Name } \\
\text { - Email } \\
\text { - Age Group } \\
\text { - Preferences } \\
\text { - Access Frequency } \\
\text { - Interests }\end{array}$ & $\begin{array}{l}\text { - Participation Level } \\
\text { - Participation Classification } \\
\text { - Knowledge Level } \\
\text { - Average Session Length }\end{array}$ \\
\hline \multirow[b]{2}{*}{ What? } & \multirow[b]{2}{*}{ Scheduled Tasks } & Subject & $\begin{array}{l}\text { - Name } \\
\text { - Pre-Requirement } \\
\text { - Difficulty level } \\
\end{array}$ & $\begin{array}{l}\text { - Difficulty level } \\
\text { - Area } \\
\text { - Reference books } \\
\end{array}$ \\
\hline & & Task & $\begin{array}{l}\text { - Name } \\
\text { - Description } \\
\text { - Kind of Activity } \\
\text { - Pre-Conditions } \\
\text { - Goal } \\
\end{array}$ & $\begin{array}{l}\text { - Activities } \\
\text { - Constraints } \\
\text { - Estimated Effort } \\
\text { - Deadline } \\
\text { - Status } \\
\end{array}$ \\
\hline When? & Completed Tasks & Historical & $\begin{array}{l}\text { - Contextual Elements used } \\
\text { to carry out the task }\end{array}$ & $\bullet$ \\
\hline \multirow{3}{*}{ Where? } & \multirow{3}{*}{ Setting } & Session & $\begin{array}{l}\text { - Duration of the session } \\
\text { - Session Classification }\end{array}$ & - Session Supervisor \\
\hline & & Environment & - Used Tool & $\bullet$ \\
\hline & & Location & - User Location & $\bullet$ \\
\hline \multirow[t]{2}{*}{ How? } & \multirow{2}{*}{$\begin{array}{l}\text { Relationship } \\
\text { between } \\
\text { People and Tasks }\end{array}$} & Interaction & \multicolumn{2}{|c|}{$\begin{array}{l}\text { - Related Task } \\
\text { - Messages Exchanged (including: author, addresses, date, time, } \\
\text { discussion subject, message subject, abstraction used, related message) }\end{array}$} \\
\hline & & Planning & - Procedures & - Strategies \\
\hline
\end{tabular}

Information about the environment where the interaction takes place (Session, Environment and Location Context) - it consists of information that characterises the environment and the current session where the interaction takes place and influences task completion. It also includes the user's location, since it can change each time that users access the environment;

Information about the relationship between people and tasks (Interaction and Planning Context) - in CSCLE it is important to know who is doing what, i.e. what is the task's execution plan and what is being discussed in the environment. This type of information is represented in two kinds of context:

a) Interaction Context - it has information about the interactions that take place in the environment and about users' behaviour when interacting;

b) Planning Context - it consists of information about the course execution plan (generally presented in the pedagogical model of CSCLE). This is the difference between the official plan (made by the teacher) and the practices developed by students when they "contextualise" the plan in order to tailor the problem solving to the context at hand. In learning, an interesting side-effect of this approach is to clearly identify when a learner goes towards a dead-end way before the learner reaches it;

The information presented in this conceptual framework (Table 1) can be captured explicitly (e.g., by asking a user information such as her/his age group or preferences) or implicitly (e.g., by using some kind of sensor or monitoring agent). The contextual information can also be available in some models or databases of the CSCLE (e.g. Task, Group and Planning Contexts can be captured in the Domain and Pedagogical Models in CSCLE).

To make matters clearer, let us consider the Individual Context. Most of the information in individual contexts can be captured explicitly, for example, by using a questionnaire that the user completes when s/he first logs in. Other information can be added to the individual context according to the behaviour of the user during the chat session. For 
example, the contextual element participation classification (see Individual Context in Table 1) classifies the user performance during the chat session. Thus, according to her/his performance, the user will be classified according to the following stereotypes ${ }^{2}$ : challenger, agreer, remiss, tutor, contributor, questioner, and inattentive. All the captured contextual information is stored in a Contextual Repository.

\section{Creating the LiM: Interaction Modelling AND STORING}

Once the interactions are structured and contextualised, they are stored in the LIM. To model the LIM, we have developed a multidimensional model. By using multidimensional modelling [23], we can apply analytical queries to explore the information stored in the model and generate feedback to both students and teachers.

Multidimensionality is based on the duality factdimensions, i.e. facts are analysed regarding data in the dimensions [23]. A fact represents a subject of analysis; i.e. something that is not known in advance, an observation in the environment at a given time (for example, quantity of tasks or number of users). While dimensions show the different points of view, we can use them to study the fact. Dimension tables contain attributes that describe fact records in the fact table.

The LIM's multidimensional model was based on the nine contextual categories (Group, Individual, Task, Subject, Historical, Session, Environment, Location and Interaction contexts) described in Table 1, for the purpose of better characterising the facts to explore (the number of interactions and the number of users). The planning context was not considered in this stage of the research. In the model, the historical context encompasses all the others and the individual context was put in the User dimension. Thus, the LIM's multidimensional model (Figure) has one fact table (with two facts: the number of interactions and the number of users) and nine dimensions (eight contextual dimensions and one time dimension). This model allows the exploration of facts according to the user's need and the context desired.

Analytical Queries can be used to explore the LIM's information. This kind of analysis can be used both by the users (directly) and by the CSCLE to support students and teachers and generate reports giving details about previous discussion and different types of learner contributions. Thus, it will be possible to answer the questions for three kinds of users: teacher, students and tutors.

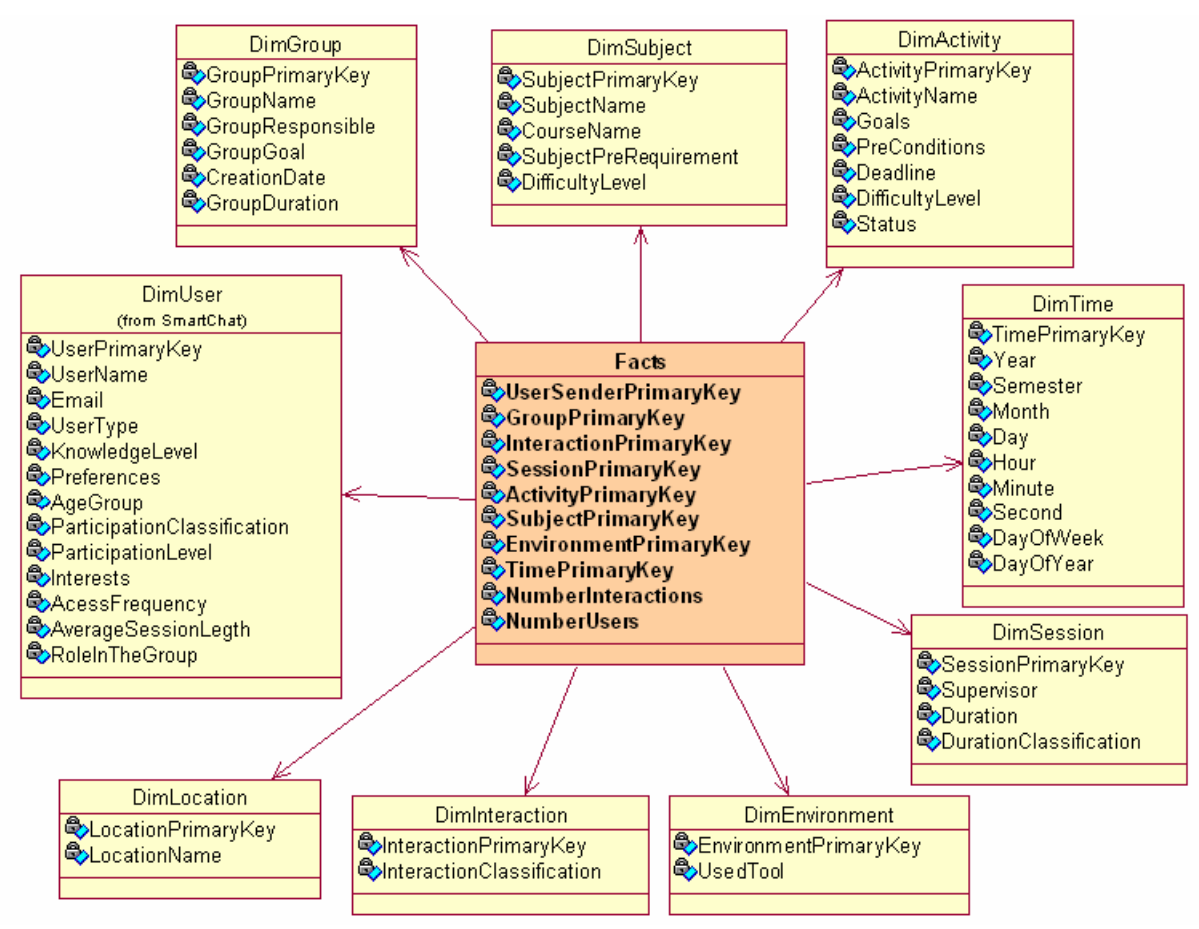

Figure 2: LIM's Multidimensional Model

\footnotetext{
${ }^{2}$ Further details on stereotype user modelling can be found in [29]
} 
Some examples of questions (obtained from discussions among Computer Science teachers and refined according to the feedback given in workshops, seminars and conferences where this work was presented) are presented below:

1) To teachers

a) What were the subjects more discussed by students (they have the most number of interactions) in a specific chat session?

b) What are the subjects which the students have more doubts in?

c) Have the students discussed more when the activity has a high difficulty level?

d) What were the activities with status $\mathrm{X}$ in the period $\mathrm{Y}$ ?

e) How many students with low knowledge level have participated in the execution of activity X?

f) Mixes of the questions above.

2) To students

a) How my participation is related to the others students' participation (number of interactions and participation level)?

b) What are the subjects which I have more doubts in?

c) Who are the students that have similar difficulties than my own difficulties (in other words, students have the most of interactions using the interaction classification QUESTION in subject X)?

d) Which were the subjects that I have discussed more?

3) To Tutors

a) Who are the students that have difficulty in a subject that I know very well?

The answers of these questions will be obtained by analysing data in the Facts Tables (Figure) with regard to data in the dimensions. For example, in question a) to teachers, we can calculate the maximum number of interactions (attribute of the table of Facts) according to the Subject dimension and with the Time dimension restricted to a specific date.

One example of using these questions is the following: suppose that one teacher have gotten the answer of the question "What are the subjects which the students have more doubts in?". According to the answer, the teacher can decide to make a review class about these subjects available in the CSCLE. In another example, suppose that when students have the answers about her/his performance (e.g. question $2 \mathrm{a}$ and $2 \mathrm{~b}$ afore mentioned for the students), s/he can decide looking for the teacher or study more or ask for help to her/his friends,

By answering these questions and generating reports about them, we intend to support students and teachers in their activities and in the reflection process. This kind of feedback generation is a very important resource in CSCLE [25][44] and it is not yet provided by most CSCLE.

\section{LIM'S IMPLEMENTATION}

To put the process of construction of the LIM into practice, we have implemented a prototype of a ContextBased Environment for Interaction Analysis called SmartChat + . To simplify matters, at this point we have limited our analysis to synchronous interactions that occur in a chat environment. This is due to the fact that such interactions are a basic component of communication in any CSCLE. The chat environment used in the prototype construction was an Intelligent Chat for Discussion called SmartChat [37].

Figure 2 shows the prototype architecture, which has two components: the chat engine called SmartChat and the Context-Based Analytical Environment. This Analytical Environment consists of:

- An Agent Society composed by the Monitor and Modeller Agents and three repositories called Contextual Repository, Domain Knowledge Base and Interaction Log. The Agent Society is responsible for structuring and contextualising the interactions as well as for recording them in the Interaction Log. A discussion session starts when the chat room is opened and the students $\log$ in. It finishes when all students log out. Moreover, the Agent Society is also responsible for acquiring all the Contextual Information (according to the Conceptual Framework for Interaction Analysis Context in the Table 1) and storing them in the Contextual Repository, as well as for generating On-Line Feedback to the environment's users. The On-Line Feedback can be one of the three following actions (based on Feedback Rules stored in the Domain Knowledge Base and on the information in the individual context, stored in the Contextual Repository):

o Send support messages to users (e.g., "Well done! You've got excellent explanations!" or "You are so laconic, wouldn't you like to participate more?"), according to the stereotype $\mathrm{s} /$ he fits in;

o Suggest references related to the subject being discussed (e.g., "If you have doubts about this 
subject, then you should consult the X book"); these references are stored in the Domain Ontology (that is part of the Domain Knowledge Base);

o Name another chat user that may collaborate with the user having difficulties (e.g., "Look, maybe Joe can help you with your doubts. Why don't you talk to him?"). In this last case, the agent looks for somebody with the "tutor" or "contributor" stereotype and that has a good knowledge level in the subject that the user is having difficulty.

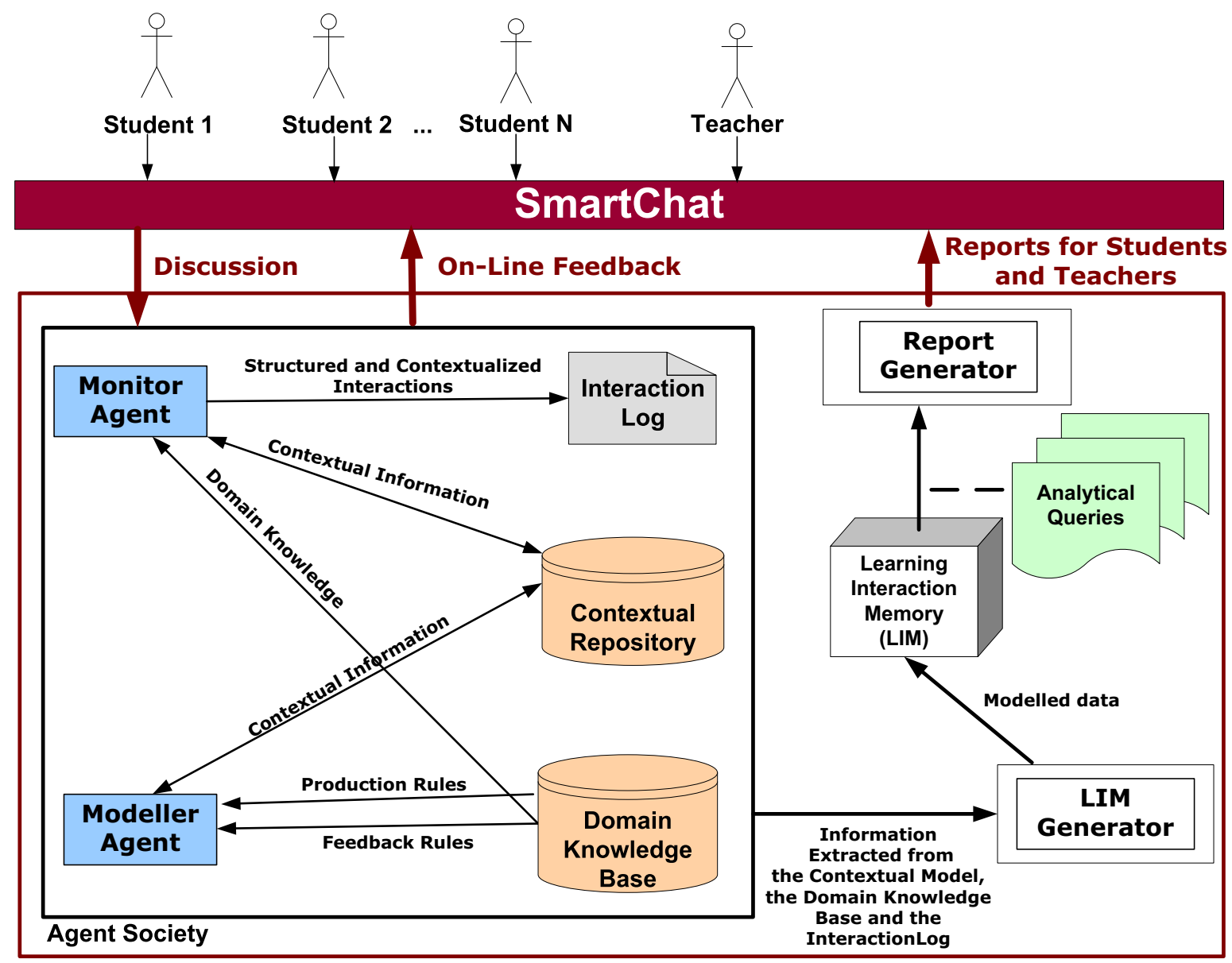

Context-Based Analytical Environment

Figure 2: SmartChat+'s Architecture

- The LIM Generator that, at the end of the session, groups the interactions related to that session (extracted from the Interaction Log), takes the Contextual Information from the Contextual Repository, models these data in a multidimensional schema (according to the LIM's Multidimensional Model in the Figure 3) and stores them in the LIM.

- The LIM is a shared organised memory that corresponds to the discussion database. It is the result of a process of accumulating data generated by group members during discussions in the chat environment and their respective context. Indeed, the historical context is in the LIM (see Table 1).

- The Report Generator is responsible for analysing the interactions (exploring the LIM) and issuing different reports for teachers and students during and at the end of the discussion session. Via this module, it is possible to generate statistical reports as well as more complete reports based on the information about previous discussions

More details about the SmartChat + can be found in [36]. 


\subsection{IMPLEMENTATION ISSUES}

SmartChat + was developed in Java [12] and uses RMI (Remote Method Invocation) [12] in the communication between server and chat clients. The agents (Monitor and Modeller) were implemented as threads in Java. The Modeller Agent uses production rules (located in the Contextual Repository) and an inference engine called JEOPS [18] to assign stereotypes to users (e.g., remiss, tutor) and also to decide the better feedback to give to students.

The individual, group, location and environment contexts are stored in the Contextual Repository. It was implemented using both XML [28] and a relational database. Indeed, there is a flag, in a properties file, to indicate where the information will be stored (in the XML file, in the database or in both at same time).

At present, the employed Domain Ontology represents the domain of Object-Orientation [41] and was defined in XML [28] due to its seamless integration with Java and the easy representation of hierarchical data structures

The LIM was modelled in a multidimensional schema and was implemented using a data warehouse structure in the DBMS (Database Management System) Microsoft SQL Server 2005 [26].

The Report Generator module generates reports to teachers and students using OLAP (On-Line Analytical Process) queries [23] to explore the information stored in the LIM. To submit OLAP queries to the LIM, the Microsoft Analysis Sever Tool was used. The tool is integrated to the Microsoft SQL Server 2005 [26] under the name SQL Server Business Intelligence Development Studio.

\section{EXPLORATORY STUDIES AND RESULTS}

This section presents the initial exploratory studies carried out with the SmartChat and SmartChat + and their obtained results.

\subsection{Methodology}

Since SmartChat + was developed in an incremental way (by gradually integrating new functionalities or modules to include a new stage of the Context-Based Process described in section 3), the exploratory studies were performed in different development stages of the SmartChat + . Therefore, we performed a series of eighteen exploratory studies divided into four groups, each one with different goals and happening at a different development stage of the prototype:

- In the first group, the prototype was only a chat engine (SmartChat) and the goal was to test its usability.
- In the second group, the Agent Society was integrated to SmartChat, forming the SmartChat (Figure 3). At that time, the Agent Society was carrying out just the first step (Interactions Structuring) of the ContextBased Process (c.f. Section 3). Thus, the goal was to test the use of the Argumentation Model abstractions and the quality of the generated Discussion Graph. Moreover the correctness of the Domain Ontology (at that time, it represented the domain "Intelligent Agents") was verified together with the quality and usefulness of an initial feedback generation.

- In the third group, the SmartChat + counted with the chat engine, the Agent Society (with the Monitor Agent, the Modeller Agent, the Domain Knowledge Base, the Interaction Log and the Contextual Repository), the LIM Generator and the LIM (see figure 3). At that time, the SmartChat + carried out the first (Interaction Structuring), second (Interaction Contextualizing) and third (Interaction Modelling and Storing) stages of the Context-Based Process for Interaction Analysis (c.f. section 3). Additionally, the on-line feedback was improved based on the information contained in the Interaction Log and in the Contextual Repository. In this version of the prototype the Domain Ontology was changed to formalise the concepts pertaining to "ObjectOrientation". The goals of the exploratory studies in this group were to check whether the contextual information captured and used by the prototype was correct and whether the storage of the information at the LIM was being done properly. Moreover, another goal was to assess the quality of the improved on-line feedback and of the statistical reports provided by the environment.

- In the last group, the SmartChat+ was completed (all stages of the Context-Based Process for Interaction Analysis were implemented) and the goal was to verify the usefulness of the generated reports with users as well as their satisfaction with the prototype.

In each group, several test sessions were carried out. In each of them, users (students and teachers) were invited to use the prototype for a period of 60 to 90 minutes, to discuss about the subject of the current class. In order to achieve the goals discussed above, the following techniques were used: interviews, questionnaires, direct observation of the users, manual analysis of the interaction log (it entails opening the log file and analysing, message by message, all the information into it (e.g., check what the most discussed subject was), manual analysis of the contextual repository (it entails opening the Contextual Repository and checking the contextual information recorded in it, item by item) and observation of the server console 
(since the system presents messages in the server console about all the operations that were been executed by the system internal components (e.g., the agents), the observation of these messages could help to check the operations in course).

Even though we have performed four sets of exploratory studies, aiming at investigating various issues as mentioned before, in this paper, we will only discuss the exploratory studies and results related to the construction, contextualization and exploration of the LIM. Therefore, in this section only three groups of exploratory studies, comprising sixteen experiments will be described in the following subsections. All the details about the complete exploratory studies can be founded in [36].

\subsection{FIRST GROUP}

In the first group of exploratory studies, the prototype neither captured nor used any kind of context and the LIM had not been implemented. Yet the prototype was already giving some on-line feedback (mostly motivational messages depending on the user classification) and generating simple statistical reports (one to the student and one to the teacher) at the end of the discussion session. The student statistical report was individual and showed her/his own performance in the discussion (e.g., the most discussed subject by her/him, how many messages s/he had sent); and the teacher statistical report had information about the students and the global discussion (e.g., both the most difficult and most discussed subjects; indication of the more accomplished learners in each subject, so that they can be used as peer tutors for students having difficulties, and a list of apathetic students who needed to be motivated).

The goals of the experiment carried out at this point were to verify: (1) the level of acceptance of the initial on-line feedback and (2) the level of acceptance and the correctness of the statistical reports produced.

To perform the test, ten undergraduate and graduate students were invited to participate in four discussion sessions about Intelligent Agents using the environment, over a two-week period (two per week). In each session, the participants, all in the same laboratory, discussed about Intelligent Agents for 90 minutes. During the discussion two people have observed users as they worked and took notes about the activity that took place in order to measure what they did (Direct Observation). At the end of the discussion, the users were asked to fill in an evaluation questionnaire about the environment. Subsequently, the answers of the questionnaire were analysed by the two observers.
The participants have liked the initial statistical reports but they proposed that the report generated must be improved to offer more information and statistics about the chat. In relation to the on-line feedback produced, the users have suggested that it could indicate references to the subjects that participants have presented some difficulty in discussing.

\subsection{SECOND Group}

In the second group of exploratory studies, the SmartChat + was carrying out the interaction contextualisation and the LIM was being created (but it was not being explored). Additionally, the on-line feedback was improved based on the information contained in the Interaction Log and in the Contextual Repository (see Figure 3). The goals of the exploratory studies in this group were to check whether the contextual information captured and used by the prototype was correct and whether the storage of the information at the LIM was being done. Moreover, another goal was to assess the quality of the improved on-line feedback and statistical reports provided by the environment.

To perform the exploratory studies, between 20 and 28 students (graduated and under graduated students) were invited to participate in ten discussion sessions about Object-Orientation using the SmartChat+. Three teachers of the Object-Orientation course were also invited to participate in these discussion sessions. In this group of exploratory studies, we carried out ten discussion sessions, over a one month period. Eight sessions were performed without the presence of any teacher and two sessions with, at least, the presence of one teacher. All the participants were allocated in the same laboratory and all sessions lasted for about 90 minutes.

To verify the correctness of the contextual information captured, the information in the Contextual Repository and in the Interaction Log was manually analysed, observing item by item each piece of information recorded. To verify the information stored in the LIM, the multidimensional database was queried and the query results compared, manually, with the information in the environments' repositories (Contextual Repository, Interaction Log and Domain Knowledge Base). Finally, to verify the level of acceptance of the feedback and reports generated, at the end of the discussion, the users were asked to fill in an evaluation questionnaire about the environment. Subsequently, the answers of the questionnaire were analysed by two researchers and the results used to improve the prototype and the generated feedback, and to guide in the creation of the new reports. 
Concerning the contextual information, the Interaction Log and the Contextual Repository were analysed and it could be checked that the contextual information was acquired correctly. Also, it was possible to confirm that the multidimensional model of the LIM was correctly filled in through the execution of queries in the multidimensional database in order to compare the results of the queries with the information stored in the repositories. All the comparison was done manually, checking line to line of the registers.

With respect to the on-line feedback, most of the users have liked it. They have said in the questionnaire that this resource had helped them to keep the focus in the discussion and to be aware about their level of participation in the discussion. Among the users that have not liked, the reason was that they have thought the feedback inconvenient. With respect to the statistical reports, the participants (students and teachers) have suggested that they should be improved to offer more information about the chat discussion in order to help the participants in their self-evaluation. They pointed out that reports including historical information and tips on how to improve one's participation in the collaborative dialogue would be useful. This would be possible with the exploration of the LIM.

\subsection{THIRD GROUP}

In the last group of exploratory studies, the exploration of the LIM was implemented in the SmartChat + and the goal of the exploratory studies was to verify the acceptance of new generated reports and their correctness.

To perform these last exploratory studies, some participants of the previous group of experiments (about 20 students and two teachers) were invited to participate of two sessions of discussion. The subject discussed was the same (Object-Orientation) in both sessions. All participants were located in the same laboratory and each session lasted for about sixty minutes. At the end of the discussion session, both the teachers and the students were asked to fill in a feedback evaluation questionnaire with questions directed at assessing the quality of the generated reports.

To verify the level of acceptance of the new format of reports the answers of the feedback evaluation questionnaire were analysed by two observers. Out of the twenty students, twelve mentioned that the reports can help them in the self-evaluation process. They have commented that it was interesting to have the possibility of seeing their behaviour in the chat compared with other users (the most and least participative user). However five students have thought that the reports were not so good because they had expected more information about other users and about themselves. Unfortunately, they have not specified what information they would like to see. With respect to the quantity of reports, some of the students have considered it enough and the others have considered it insufficient, whishing that there were more reports. This is due to the fact that it was not possible to generate a large variety of reports in the context of this work. However, considering that all the interactions and their contextual information are stored in the LIM, new queries can be created at any time.

Teachers have indicated that they have liked the reports and have mentioned that the reports could be useful to keep up with the students' behaviour in the discussion sessions. The information in the reports could help them to choose the better way to guide the users and give some feedback to them. They have also mentioned that they do not know other chat that offers such kind of reports. Moreover, the teachers have suggested that new information should be included in the reports: information about a group or individuals (e.g., email, interests and knowledge level), students' location when interacting in the discussion session and historical evolution of the user classification. The teachers have also asked us to include the possibility of saving the reports in PDF format in the system.

\subsection{Discussion}

All the exploratory studies (with their settings, goals and results) are summarized in Table 2. It is important to observe that the analytical queries have made easier to explore the contextualised historical information stored in the LIM to generate reports for students and teachers. Moreover, the contextual information acquired has helped to generate more complete reports and more qualitative feedback (e.g., indicating the classification of the user according to the participation level). 
Table 2: A Summary of the Exploratory Studies

\begin{tabular}{|c|c|c|c|c|}
\hline \multicolumn{2}{|r|}{ Exploratory Studies } & Group 1 & Group 2 & Group 3 \\
\hline & Number of Students & 10 & 20 to 28 & 20 \\
\hline \multicolumn{2}{|c|}{ Number of Teachers } & 1 & 0 to 3 & 2 \\
\hline \multicolumn{2}{|c|}{ Number of Discussion Sessions } & 4 & 10 & 2 \\
\hline \multicolumn{2}{|c|}{$\begin{array}{r}\text { Session Duration } \\
\end{array}$} & 90 minutes & 90 minutes & 60 minutes \\
\hline \multirow{4}{*}{ 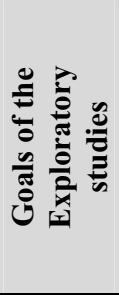 } & $\begin{array}{l}\text { Capture of the } \\
\text { Contextual Information }\end{array}$ & & $\mathrm{X}$ & \\
\hline & LIM's Generation & & $\mathrm{X}$ & \\
\hline & $\begin{array}{l}\text { Acceptance of the On- } \\
\text { Line Feedback }\end{array}$ & $\mathrm{X}$ & $\mathrm{X}$ & $\mathrm{X}$ \\
\hline & $\begin{array}{l}\text { Acceptance and } \\
\text { Correctness of the } \\
\text { Generated Reports }\end{array}$ & $\mathrm{X}$ & $\mathrm{X}$ & $\mathrm{X}$ \\
\hline \multirow{6}{*}{ 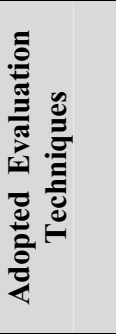 } & Questionnaire & $\mathrm{X}$ & $\mathrm{X}$ & $\mathrm{X}$ \\
\hline & Direct Observation & $\mathrm{X}$ & & \\
\hline & $\begin{array}{l}\text { Manual Analysis of the } \\
\text { Interaction Log }\end{array}$ & $\mathrm{X}$ & $\mathrm{X}$ & \\
\hline & $\begin{array}{l}\text { Manual Analysis of the } \\
\text { Contextual Repository }\end{array}$ & & $\mathrm{X}$ & \\
\hline & Queries to the LIM & & $\mathrm{X}$ & \\
\hline & $\begin{array}{l}\text { Observation of the } \\
\text { Server Console }\end{array}$ & $\mathrm{X}$ & $\mathrm{X}$ & \\
\hline \multirow{4}{*}{$\stackrel{\mathscr{0}}{\bar{\Xi}}$} & Contextual Information & & $\begin{array}{l}\text { Information captured } \\
\text { and stored correctly }\end{array}$ & \\
\hline & $\begin{array}{l}\text { Information stored in } \\
\text { the LIM }\end{array}$ & & $\begin{array}{l}\text { Information modelled } \\
\text { and stored correctly }\end{array}$ & \\
\hline & $\begin{array}{l}\text { Acceptance of the On- } \\
\text { Line Feedback }\end{array}$ & $\begin{array}{c}\text { Several suggestions } \\
\text { and critiques }\end{array}$ & $\begin{array}{c}\text { Good acceptance and } \\
\text { few critiques }\end{array}$ & $\begin{array}{c}\text { Good acceptance and some } \\
\text { suggestions }\end{array}$ \\
\hline & $\begin{array}{l}\text { Acceptance of the } \\
\text { Reports }\end{array}$ & Poor reports & $\begin{array}{c}\text { Good Acceptance and } \\
\text { suggestions of new } \\
\text { reports }\end{array}$ & Good Acceptance \\
\hline
\end{tabular}

The obtained comments from users (through interview and questionnaire) have indicated the usefulness of the generated reports (which contain the answers to the questions listed in section 5) and feedback. Indeed, most of the students have accessed the reports and they have written in the questionnaire that they liked both to have a way to get information about their behaviour in the discussion as well as to have some way to compare their behaviour with others. Similarly, teachers have considered it really useful to have more information about their students and to have the possibility of accessing summarised reports about the student's behaviour in the discussion sessions. They have indicated in the questionnaires that these reports could help them in assessing and observing the students interaction and individual progress.

\section{Conclusions And Future Work}

Although several works consider reflection as one of the most useful skills during the learning process [20][21][46], most recent CSCLE still lack ways of supporting learners' and teachers' needs and the reflection process. Our work aims to attenuate these gaps by proposing a contextualized process for Interaction Analysis that intends to support both students and teachers via the exploration of the LIM.

In this paper, we have presented the stages of our process for Interaction Analysis that focus on the creation of a LIM where contextualised interactions are modelled and stored. This LIM is a multidimensional repository built with the purpose of facilitating the execution of analytical analysis, in order to generate support for students and teachers and many kinds of feedback reports. Also, the information generated through the analysis of the data in the LIM could be used to improve and support awareness [15][42] in CSCLE. Awareness can be used to facilitate effective group communication and coordination.

One major advantage of our approach is that it considers the context where the interactions occur, creating the LIM with contextualized historical information about discussion sessions, in order to generate feedback for both students and teachers. This is an advantage because, in many cases, only the context 
can provide the full understanding of many interactions, actions or events and the correct cues to allow the correct analysis of the interactions, in order to better support user reflection and to provide adequate feedback.

The implementation and experimentation of an intelligent environment for collaborative discussions called SmartChat + was also described. This implementation has allowed us to evaluate the construction and exploration of the LIM. Moreover, the results of the experimentation were discussed, indicating the feasibility of the proposed process and its potential to support effective learning.

During the development of this work, some limitations were found. First, although the ContextBased Process for Interaction Analysis had been defined to CSCLE, a CSCLE was not used in the prototype implementation, in order to simplify matters. Instead, we have used a tool for synchronous communication among users in the SmartChat + construction. The prototype did not consider the planning context (because this context should be captured in the Pedagogical Model Knowledge Base of the CSCLE which has information such as learning strategies and course planning). The number of generated reports is still small. However, since the model of the LIM was defined and the module to load it is fully functional, new reports and queries can be constructed in the future.

Our future research will concentrate on better exploring the analytical queries and the information stored in the LIM. For example, constructing more report templates using analytical queries. We will also perform more exploratory studies with users in order to verify the usefulness of the generated reports for students and teachers. We will also work on defining a communication interface to integrate the SmartChat + to other environments (e.g. CSCLE). Additionally, we intend to use a mechanism of inference to discover knowledge from the data in the LIM, in order to try to answer the question "Why?" described in [3].

\section{REFERENCES}

[1] R. Araujo, P. Brézillon. Reinforcing Shared Context to Improve Collaborative Work. Revue d'Intelligence Artificielle. Special Issue on Applying Context Management. S. Schultz, T. Roth-Berghofer \& P. Brézillon (Eds.), 19(3): 537556,2005

[2] M. Bazire, P. Brézillon, Ch. Tijus. Eléments Intervenants dans le Contexte d'une Activité Finalisée. Ed. J.M.C. Bastien. In Actes des Deuxièmes Journées d'Etude en Psychologie
Ergonomique - EPIQUE'2003, INRIA, pages 281-286, 2003.

[3] M. Bazire, P. Brézillon. Understanding Context before to Use It. In Proceedings of Modelling and Using Context (CONTEXT-05), A. Dey, B.Kokinov, D.Leake, R.Turner (Eds.), Springer Verlag, LNAI 3554, pages 29-40, 2005.

[4] M. R. S. Borges, J. A. Pino. Requirements for Shared Memory in CSCW applications. In Proceedings of 10th Annual Workshop On Information Technologies and Systems (WITS'2000), Brisbane, Australia, pages 211-216, 2000.

[5] P. Brézillon. Context in Problem Solving: A survey. The Knowledge Engineering Review, 14(1): 1-34, 1999.

[6] P. Brézillon. Making Context Explicit in Communicating Objects. Eds: C. Kintzig, G. Poulain, G. Privat, P.-N. Favennec, Communicating Objects, Hermes Science Editions, Lavoisier, 2002.

[7] A. Collins, J. S. Brown. The Computer as a Tool for Learning through Reflection. Eds. H. Mandl and A. Lesgold. Learning Issues for Intelligent Tutoring Systems, Springer-Verlag, New York, pages 1-18, 1988.

[8] E. J. Conklin. Designing Organizational Memory: Preserving Intellectual Assets in a Knowledge Economy.

http://www.touchstone.com/tr/wp/DOM.html. June, 2007

[9] M. Constantino-González, D. Suthers. Coaching Collaboration by Comparing Solutions and Tracking Participation. Eds. P. Dillenbourg, A. Eurelings \& K. Hakkarainen. In Proceedings EuroCSCL 2001, Maastricht, The Netherlands, pages 173-180, 2001.

[10] J. Coutaz, J. L. Crowley, S. Dobson, D. Garlan. Context is Key. Communications of the ACM, 48(3): 49-53, 2005.

[11] T. Daradoumis, A. Martínez-Monés, F. Xhafa. A Layered Framework for Evaluating On-line Collaborative Learning Interactions. International Journal of Human-Computer Studies, 64(6): 622635, 2006

[12] H. M. Deitel. Java - Como Programar. PrenticeHall do Brasil, 6 ${ }^{a}$ edição, 2006 (in Portuguese).

[13] A. K. Dey, D. Salber, G. D. Abowd. A Conceptual Framework and a Toolkit for 
Supporting the Rapid Prototyping of ContextAware Applications. Context-Aware Computing, Human-Computer Interaction (HCI) Journal, 16: 97-166, 2001

[14] P. Dillenbourg. What do You Mean by Collaborative Learning? Ed. P. Dillenbourg. Collaborative-learning: Cognitive and Computational Approaches, Oxford: Elsevier, pages 1-19, 1999.

[15] P. Dourish, V. Bellotti. Awareness and Coordination in Shared Workspaces. J. Turner and R. Kraut (Eds.) Proceedings of the conference of the Computer Supported Cooperative Work (CSCW'92). ACM Press, Toronto, Ontario, pages 107-114, 1992.

[16] P. Dourish. What We Talk About When We Talk About Context. Personal and Ubiquitous Computing, 8(1): 19-30, 2004.

[17] M. E. Eleuterio. Mediating Collective Discussions Using an Intelligent ArgumentationBased Framework. In Proceedings of World Conference on Educational Multimedia, Hypermedia and Telecommunications 2002, Chesapeake, VA: AACE, pages 472-477, 2002.

[18] C. Figueira Filho, G. Ramalho. JEOPS - Java Embedded Object Production System. Eds Monard, M. C; Sichman, J. S. In Proceedings. Lecture Notes in Computer Science, 1952: 53-62, Springer-Verlag, 2000.

[19] H. Fuks, M. L. Cunha, M. A. Gerosa, C. J. P. Lucena. Using the AulaNet Learning Environment to Implement Collaborative Learning via Internet. Innovations 2003 - World Innovations in Engineering Education and Research. INEER, USA, pages 225-235, 2003

[20] B. Goodman, A. Soller, F. Linton, R. Gaimari. Encouraging Student Reflection and Articulation using a Learning Companion. International Journal of Artificial Intelligence in Education, 9(4): 237-255, 1998.

[21] J. Grundy, W. Mugridge, J. Hosking, R. Amor. Support for Collaborative, Integrated Software Development. Ed. Verrall. In Proceedings of Software Engineering Environments, IEEE CS Press, Noordwijkerhout, Netherlands, pages 8494, 1995.

[22] M. Kantor, B. Zimmermann, D. Redmiles. From Group Memory to Project Awareness Through Use of the Knowledge Depot. In Proceedings of the 1997 California Software Symposium, UCI
Irvine Research Unit in Software, Irvine, CA, pages 19-26, 1997.

[23] R. Kimball, L. Reeves, M. Ross, W. Thomthwaite, The Datawarehouse Lifecycle Toolkit: Tools and Techniques for Designing, Developing and Deploying Data Warehouses. Jonh Wiley \& Sons, New York, 1998

[24] W. Kunz, H. Rittel. Issues as Elements of Information Systems. Working Paper No. 131, Institute of Urban and regional Development, University of California, Berkeley, 1970.

[25] J. Lonchamp. Supporting Synchronous Collaborative Learning: A Generic, MultiDimensional Model. Computer-Supported Collaborative Learning. 1(2):247-276, 2006

[26] Microsoft SQL Server - Analysis Server. http://www.microsoft.com/sql/default.mspx November, 2006.

[27] M. K. Pinheiro, J. V. Lima, M. R. S. Borges. A Framework for Awareness Support in Groupware Systems. Computers in Industry, Netherlands, 52(1): 47-57, 2003.

[28] E. T. Ray. Learning XML. O'Reilly and Associates, 2001.

[29] E. Rich. Stereotypes and User Modeling. Eds. A. Kobsa \& W. Wahlster, User Models in Dialog Systems, Springer-Verlag, Berlin, Heidelberg, pages 35-51,1989.

[30] M. Rittenbruch. ATMOSPHERE: A Framework for Contextual Awareness. International Journal of Human-Computer Interaction, 14(2): 159-180, 2002.

[31] J. Robertson, J. Good, H. Pain. BetterBlether: The Design and Evaluation of a Discussion Tool for Education. International Journal of Artificial Intelligence in Education, (9): 219-236, 1998.

[32] M. G. P. Rosa, M. R. S. Borges, F. M. Santoro. A Conceptual Framework for Analyzing the Use of Context in Groupware. In Proceedings of International Workshop on Groupware, Autrans, France, Lecture Notes in Computer Science, Berlin, Germany, 2806: 300-313, 2003.

[33] F. M. Santoro, P. Brézillon. The Role of the Shared Context in Group Storytelling. Computers and Informatics, Special Issue on "Groupware, Context and Design”. M.R.S. Borges, H. Fuks, S. Lukosh and J.A. Pino (Eds.) 25(6): 1001-1026, 2006. 
[34] J. W. Satzinger, M. J. Garfield, M. Nagasundaram. The Creative Process: The Effects of Group Memory on Individual Idea Generation. Journal of Management Information Systems, 15(4):143-160, 1999.

[35] P. Seetharaman, S. Paul. Organizational Memory in Group Decision Making: Use of Constructive Group Conflict. In Proceedings of the 11th European Conference on Information Systems (ECIS), pages 19-21, 2003.

[36] S. A. Siebra. Analysing Participant's Interactions in Collaborative Learning Environment. CIn Federal University of Pernambuco, PhD. Thesis, Recife - Pernambuco - Brazil, 2007.

[37] S. A. Siebra, C. R. Christ, A. E. M. Queiroz, P. C. A. R. Tedesco, F. M. Barros. SmartChat - An Intelligent Environment for Collaborative Discussions. Proceedings of ITS 2004, Maceió, AL, pages 883-885, 2004.

[38] S. A. Siebra, A. C. Salgado, P. A. Tedesco. Structuring Participants' Interactions in Collaborative Learning Environments. Proceedings of WebMedia/LA-Web 2004 $W C S C W$, Ribeirão Preto, São Paulo, Brasil, pages 181- 187, 2004.

[39] S. A. Siebra, A. C. Salgado, P. A. Tedesco, P. Brézillon. Identifying the interaction context in CSCLE. Modeling and Using Context (CONTEXT-05), A. Dey, B.Kokinov, D.Leake, R.Turner (Eds.), Springer Verlag, LNAI 3554, pages $464-475,2005$

[40] Siebra, S., Salgado, A.C., Brézillon, P. and Tedesco, P. A Learning Interaction Memory using Contextual Information. In: Context and Work Group Workshop, 2005, Paris. Context and Work Group Workshop, 2005. vol. 133, pages 112,2005

[41] A. Sintes. Aprenda Programação Orientada a Objetos em 21 dias, Makron, 1a. edição, 2002 (in Portuguese)

[42] M. Sohlenkamp. Supporting Group Awareness in Multi-User Enviroment Through Perceptualization, M. Sc. Dissertation., Fachbereich Mathematik-Informatik der Universit - Gesamthochschule - Paderborn, 1998.

[43] A. Soller, A. Lesgold. A Computational Approach to Analyzing Online Knowledge Sharing Interaction. In Proceedings of Artificial Intelligence in Education 2003, Sydney, Australia, pages 253-260, 2003.
[44] A. Soller, A. Martínez-Monés, P. Jermann, M. Muehlenbrock. From Mirroring to Guiding: A Review of State of the Art Technology for Supporting Collaborative Learning. International Journal of Artificial Intelligence in Education, 15(4): 261-290, 2005.

[45] SPEM-OMG, The Software Process Engineering Metamodel Specification. Version 1.1, January 2005.

http://www.omg.org/technology/documents/mode ling_spec_catalog.htm\#SPEM June, 2007)

[46] P. A. Tedesco. MArCo: Building an Artificial Conflict Mediator to Support Group Planning Interactions. International Journal of Artificial Intelligence in Education. No. 13, pages 117-155, 2003

[47] A. L. Veerman. Computer-Supported Collaborative learning through argumentation, PhD. Thesis, University of Utrecht, 2000.

[48] A. C. H. Vieira, L. Teixeira, A. Timóteo, P. Tedesco, F. Barros. Analyzing On-Line Collaborative Dialogues: The OXEnTCHÊ-Chat. In Proceedings of 7th International Conference on Intelligent Tutoring Systems (ITS'2004), Maceió - Brasil, pages 315-324, 2004.

[49] J. P. Walsh, R. D. Dewar. Formulation and the Organizational Life Cycle. Journal of Management Studies, 24(3), 1987. 